\title{
TO THE THEORY OF SYSTEMS: A BRIEF LOOK AT THE UNDERLYING OF NOTIONS IN THE FIELD OF CONCEPTUAL CONTENT
}

\author{
VLADIMIR P. KOLYADA
}

\begin{abstract}
From the systems theory point of view the attempting was made to establish the most suitable logical and semantical conceptual definition for systems notions while maintaining the maximal compatibility in the theoretical field, which enable to bring together systems with any scale and complexity to a single strategy of interpretation of their dynamics. The special semantic clarifications for notions which generating theoretical ground were established. Thus specified fundamental and system-forming processes. Direct relations of specific terms and subtleties of differences of system notions, their roles for creating solid theoretical representation were highlighted. Some examples of the biochemical types are given. An explicit, semantic clarification of some concepts describing the dynamics of systems and states is proposed.
\end{abstract}

Keywords: theory of systems, notions, universal, evolution.

\section{INTRODUCTION}

Understanding system-forming phenomena represents itself a highly important field of study, which allows to establish true mechanisms of genesis, development, functional regularity and self-organization of world-perceived systems on the fundamental level, particularly non-linear dynamic systems. Systems approach suggests not just mathematical modeling of some approximately isomorphic systems (whose dynamics are compatible or almost compatible with a certain differential equation) but primarily comprehensive determination and interpretation systems notions that composing itself the essence of explanations for regularity in the theory of systems. Based on exhaustive logical and semantical conclusions in regard to notional definition that composing the units of metalinguistic concept for current issue, we will attempt to specify possible discrepancies in interpretation of systems dynamic from the point of view of system-based phenomena wherein deviating from specific models (restrictions) or establishing compatibility of corresponded logic with as much as possible described systems, at least in hypothetical field. A prerequisite is made for implementation of a general strategy for defining a system without ignoring specialization i.e. avoiding particular mathematical models on the one hand and statistical analysis methods, which is not allowing to fully implement the quality compatibility of the systems under consideration, on the other.

\section{SUBSTRATUM (ENVIRONMENT)}

The basis on which a function can be implemented can be considered as an environment. Independently of how the level of abstraction the realization was caused, 
the material movement (according to the existing interpretation of modern science) eventually happening as the initiator of the function. For the convenient reason the set of all spaces can be named a real environment, defining as a universal $U$ and described as follows:

The real environment in system understanding primarily corresponds with the conceptual definition of the active environment that in the inhomogeneous space of which, relatively $(\dagger)$, stationary states exist (sustainable active environments on a spatial scale) the dynamics of which are caused by special, local in relation to such systems, constraints (impulses, disturbances, or vice versa, adynamic iterations). The real substratum or environment has three major spaces material (physical) $M$, functional $P$ and also informational $I$, when the material substrate is identified to the state of the system, which behaves in accordance with the natural (physical, chemical, etc.) constraints of environment when $U \equiv M^{\frac{n}{m}}+P^{n}+I^{n}, \quad M^{\frac{n}{m}} \supseteq P^{n} \supseteq I^{n}, \quad M^{\frac{n}{m}}: M^{\frac{n}{m}} \rightarrow f\left(P^{n}\right): P^{n} \rightarrow I^{n}$ provided $n(M, P, I), m \rightarrow \infty$, where $n$ - dimension of action space, $m$ - coefficient of restrictions. In accordance with the systems approach and continuity problems, the physical space can be formally identified with the functional space, when the concepts of movement and action in a logical-semantic sense describe the same observable phenomenon on some continuum set.

The functional environment (functional space) corresponds to an abstract ideally isomorphic projection of the material substrate and expressed in the degree of negentropy or its functional ordering. Informational environment (information or signs space), is an abstract ideally isomorphic projection of the functional and the material substrate accordingly and expressed in the degree of negentropy or its informational order. Thus, is highlighting a separate class of functional and information systems that exist at a certain abstract level and are a consequence of entropy-negentropy (chaos $\dagger$ ) or a form (structure) of material substratum being at the same time absolutely isomorphic to the material substrate. The environment is a set of systems ideally isomorphic to each other, simultaneously acting in qualitatively different spaces when the unity of the environment is expressing through the isomorphism. Therefore, in the process of analyzing a certain system, often, the entire axis of related systems can be analyzed simultaneously.

An important characteristic of the functional environment is the tendency to change its functional concentration from state to state (in the process of movement) or analyzing from the perspective of the information environment a change in the information order. In other words, a functional substrate is more functional than it is more ordered and more addicted to implement informational order or information synthesis. It should be noted that the orderliness and stability of the environment are not identical notions - orderliness tends to predetermine sustainability, in turn, stability tends to negative orderliness.

The unity or atomicity of the real environment is caused not only by its internal isomorphic nature but also by the presence of cyclical conditioning of its material, functional and informational components whose existence is possible only in their equal dependence on each other. For example, in the absence of a functional or informational component, the substrate would be in the first case a model with no dynamics, changes, movement, and therefore progressive exis- 
tence. In the second case, the absence of communication possibilities between the states generating the signal. This absence inevitably leads to a constant increase of the entropy limit on any scale of the system and, to the progressive existence of a real substrate accordingly.

As the environment moves and the functional concentration fluctuates, the phenomenon of sustainability is formed inevitably and obviously predetermining fundamental evolutionary movement whose dynamics have multiple progressive polymorphism. It is also obvious that there is a phenomenon that predetermining different behavior of the local environment while maintaining a general selfsimilarity of action to some scale when system dynamics are described by local characteristics ignoring the set of lower order using summation and upper order using instantiation respectively. Such a pattern in the environment naturally indicates the occurrence of attenuation of the dependence between actions in the space of functions, when the index of functional importance decreases as the action vector moves away from a function to a certain target state with minimal importance. The scopes of such an "egocentric" set in the space of functions that highlights the transition area of the function to the external environment and losing its quality importance, defines the concept of the visibility limit for a certain functional system.

The visibility limit of the system corresponds to the length of the vector or scale of the system that the functional particular properties of which beyond its boundaries no longer matter. From the point of view of the physical environment, the visibility limit actually corresponds to the spatial geometric structure of the system (which has fairly clear functional ambits).

\section{RESTRICTIONS}

It is important to note that the limitations that synthesize the deterministic probabilistic characteristics of the system also constitute the essence of any state and action. States and actions are the executive tools in the space of the environment or phenomena that form the dynamics while restrictions are a direct determinant factor. At the same time, all phenomena synthesized by environmental restrictions certainly are cross-cutting and therefore interact at different levels with different recursiveness and synthesized (including itself) probabilistic factors. We emphasize that the concept of restriction means a non-classical increase in the "rigidity" of the system's dynamics due to the transformation or replacing the functions of local sets and accordingly devoid the degree of freedom of a limited system. The consequence of the conditional absolute removal of restrictions from a certain system, obviously will be the primitivization of the system's structure by devoid it of the corresponding fundamental properties which predetermine the dynamics of such a system in the environment. Formally, we can say that the restriction $r$ corresponds to the function (attribute) of the set $n$ of some system $S$ in the material environment, when $r=f(n) \subset S$.

\section{NOISE AND CHAOS}

The concept of noise and chaos in a semantic sense have common features with the only difference that noise is apparently a special case of chaos and closely 
related to the concept of dispersion, that is, a phenomenon that has a certain source or beginning the gradual concentration of which differs as it moves, if spatial restrictions contribute to this. The presence of noise in some state naturally implies the existence of a source of such noise and thereby defining the element of noise as part of the gradual dispersion as it moves, in the distal direction, functional and information uncertainty increases (Lyapunov exponent). It can be considered that in relation to another system such an element of noise contains less of functional importance. To achieve a scale of uncertainty that goes beyond the visibility (when turbulent flows form signs of homogeneity beyond the scale) the set of such dissipation can correspond to the criterion of chaost.

Thus, the only source of increasing uncertainty and negative ordering in the real environment is the phenomenon of noise (or intersection of vectors $V_{n}$ of the functional state $A$ of the set $a$ in the state $B$ of the set $b$, when

$$
V_{a} \subset A_{a}=\sum_{k=1}^{n} a_{k}, V_{b} \subset B_{b}=\sum_{k=1}^{n} b_{k}, V_{a} \cap V_{b},
$$

at $n \rightarrow \infty$ in continuity) coming from some state, that is the system or state is a source of noise the dispersion dynamics of which generates a functional "pattern" constituting the topological essence of the system. This is not directly about the scenarios of the chaos appearance eg Ruelle-Takens, Pomeau-Manneville or Feigenbaum as noise generators, but about the structure of the dynamics in the physical environment, where each state is an element of noise regardless of whether such a state moves (on a certain scale) with a chaotic potential or not. An example of an elementary formal representation of the appearance of homogeneity (an increasing uncertainty) is any $2 n$-dimensional model on the Poincaré map with increasing energy (eg Henon-Heiles model) [1]. It is worth to noting that isomorphic interpretations of such a phenomenon are undoubtedly relevant in the functional and information spaces of any level of the hierarchy and probably play a key role in understanding the origin of instability initiation.

The more distally the vector (action) of such a "pattern" is from its central structure, the more its properties tend to be interpreted as the notion of the randomness phenomenon (or increasing complexity as going beyond visibility) and the less likely it is to form under pressure of restrictions. Obviously, with the removal sources of noise step-by-step from the environment, primarily localized "strange" (chaotic) attractors, it is possible to achieve complete determination of any system. It seems that the reduction of restrictions increases the tendency to acquire geometric invariance or self-similarity and fractal similarity of the real environment in scale.

Entropy-negentropy, mainly and probably the only way, comes from inside the state as functional and informational signals having a destructive or, on the contrary, stable potential and further, as the scale increases, generates other state models thus forming the essence of the system topology in the substrate.

The noisiness of the environment, as a result of restrictions, creates a probabilistic $\uparrow$ situation in the environment that forms an effective or progressive functional substrate. The probabilistic situation in a logical-semantic sense is probably identical to the quantitative measure (density) of the environment on a scale. The probability of state synthesis is determined which somehow or another enters into a dependent relation to the presence of a certain (e.g. high or low) probability 
of synthesis of another set which may also be included in other probabilistic dependencies, etc. Thus, the hierarchical synthesis of states and their dynamics in a probabilistic field, which in turn is a derivative of noise in a substrate, is selfregulated.

There is an important feature of radiation in any state that can be observed, when the noise pattern spreads the worse, the more the importance and specificity of the system in which this pattern diffuses. Due to the fact that any state has an atomic form, (when all vectors that nested in it are strictly determined by restrictions) the concept of state radiation is an abstract interpretation in the sense that there is no empty space for any other vector to enter, due to their continuity or infinite density. The notion of the absence of empty space in the state is interpreted as an estimation in a quasi-space that describing the possibility of replacing one set by another if it were possible. In this case, it is impossible to replace the noise distribution vector in a certain target state.

Dynamics in quasi-space during vector $V_{n}$ assimilation can form situations (properties of restrictions) when the formations $V_{n+1} \rightarrow V_{n}$ are not possible due to congestion of the functional space at the same time, entry into the state is possible only after free up a certain functional place in the state. It should be noted that the concept of congestion is compatible only in the case of higher abstractions or abstractions that have more than one functional space for their implementation, unlike a functional environment that has one implementation space (material environment) when the importance and its properties are defined. Biochemical example of a similar phenomenon in general can be the mechanisms of proteins repressors regulation $[2,3]$.

\section{SYSTEM AND STATE}

Previously, the following conceptual formulation of the system and state should be clarified: the single iteration is a state, the system is at least one state, the system forms at least one state.

Any system $S$ is a determination of some emerging state by means of incoming action vectors $S\left(V_{n}\right) \rightarrow S^{\prime}(S)$ and, as a result of this determination, the possibility of influencing or regulating the final state or the vector itself leading to such a state $S^{\prime}(S) \rightarrow S\left(V_{n}\right)$. Individual cases of such mechanisms are clearly represented for example in protein machines, in the regulation of gene expression and lie in the ability to regulate such expression differentially, selectively controlling the expression of the type of a particular gene or protein and its amount [4]. In supercomplex systems, such regulation is already expressed in the pre-psychic and mental apparatus of the regulation of behavior (movement).

Effective functional movement is a higher order shift of the environmental functional structure relatively to the material set, with such a degree of preservation of this functional structure that will also preserve its informational row, at least, in the visibility limit of the systems related to it, while the primary (lower level) structure of the environment is certainly not capable to moving without moving its isomorphic material structure. An example of a functional structure shift, basically, without qualitative preservation of the information signal on the scale, is the shift of the reading position by one or two nucleotides in the molecu- 
lar gene apparatus that is the transition to the second and third functional space. In this case, it is possible both the preservation and the loss of functional translational consequences.

A similar effect occurs during the shift in higher functional fusion processes when two or more functional structures that are shifted into each other assimilating into one unitary structure (it can be considered that such a union occurs when after a merging the final function subsequently generates a signal identical to the function signals, as if they had not merged). Classical isomorphic phenomena in a probabilistic field can correspond to the formations of points or intersections of trajectories in the phase space at the cross-section of an invariant tori set for Hamiltonian systems.

It is necessary to define the concept of the importance of the state. The state importance degree of a set also has a rather abstract concept and in fact, it represents the entropy properties of a state in combination with the functional and informational sets that are the components of such a state or determining it also forming ordering and, as a result, stability. In turn, a state with a sufficient number of destructive signals is likely to be tend to the progression of negative ordering and negative importance accordingly. A suitable example of importance in a biochemical scale substrate is highly conserved receptor domains with a high degree of autonomy, for example, steroid or other hormones or proteins, collectively characterized by a high degree of homology, both at the level of the material substrate, when the amino acid sequence is the same or different and does not affect functionally, and at the level of the functional information environment when sequences perform a single function (for example a DNA binding sites) [5]. On the contrary, amino acid receptor sequences with a low degree of homology may have low conservative, highly specialized, highly dependent, material and functional information indicators, respectively. On the contrary, amino acid receptor sequences with a low degree of homology may have low conservative, highly specialized, highly dependent, material (amino acid sequence varies) and functionalinformation (carry out various or closely related functions, or the functional ability is insignificant on the scale) indicators, respectively. It is important to emphasize that at the same time similar properties are preserved even within different taxonomic categories, which may indicate constellation genesis in duplicating such a material and/or functional (with isomorphic topology and different material structure in the functional case) structure raised under the restrictions of a similar strategy (algorithm), or indicate congenitality for a specific ancestral gene, due to the fact that quite often closely related proteins performing similar functions have a common genetic ancestor or at least these genes were more or less homologous.

Importance in the information substrate can also be interpreted as a measure of the functional state stability. An example of informational destruction in a biological substrate can be the final death of the immune system B-cell with unsuccessful V-D-J genes functional recombination forming the $\mathrm{H}-$ and L- antigen chains [6] which is in the role of the functional essence of the cell without the possibility of restructuring its purpose with no changing the scale, when entering into another information vector at the current scale is impossible and therefore the cell is phagocytosed through certain mechanisms dissolving in the environment. Thus, the system is fragmented to a certain steady state where an attempt will be made to reach the target state in fragments in accordance with the established 
restrictions. Such of destruction can be a particular example of the fluence phenomenon and finiteness of importance.

From the informational point of view, the degree of the functional structure importance predetermines, apparently, the saturation of signal or saturation can probably be called as the amount of importance, that is, the number of vectors and especially important vectors included in the signal and thus determining the degree of importance of such a signal.

It should be noted specialization and variable properties of importance. Previously, we shall to determine an evolutionary peak notion, which is based on the synthetic theory of evolution and synergetic, can be a system that is stable on long evolutionary periods of time and corresponds to the maximum-specific categorization of matter. For example, in life, this corresponds to the categories of the species and subspecies.

A distinctive feature of systems that are more susceptible to the evolutionary peak from less susceptible systems is the specialized feature of the direction of the signals of such states or their inclusion in certain significant systems specific for such a state (cycles, constellations, regulation, etc.). In this case, for example in multicellular organisms, many unstable cells that have undergone degeneration that destabilize the microprograms and accordingly the higher programs in which they belong. The special and, on the contrary, universal functions of such a set depend on variable factors that determine the importance of individual, functional-information programs that run such cells. There are special factors that have many dependencies, which in turn have their own algorithms for regulating the importance and when changing their dynamics (self-destruction) they are capable of much more disturbing the main program, in contrast to a large number of factors with insignificant importance, which is natural. In relation to the selfdestruction, it should be clarified that states with high importance (orderliness) tend to be stable but also, the occurrence of a destructive signal that capable of destabilizing and destroying such a state is permissible.

There is an important pattern in the environment - function-dependent states, as a rule, tend to convergence $\dagger$ or reduce the distance of signal communications as effectively as possible and of course the intensity of such a phenomenon is the higher, the closer a state is localized to a certain evolutionary peak. Due to such a convergence, only and only the function of the highest order $f^{\prime}(x)$ of the formed system $P$ corresponds to the informational purpose $I$ of the same order $I\left(f^{\prime}(x)\right)$, because the final function is the limit functional-information structure $G_{P I}$ of a certain scale of the set $U \supset G_{P I}$ and can be performed only on such a specific scale:

$$
G_{P I} \approx f^{\prime}(x)_{n+1}=f\left(G_{P}\right): G_{P} \rightarrow G_{I}, \quad \exists T \subset G_{P I} \rightarrow f^{\prime}(x)_{n+1}, T \approx \sum_{k=1}^{n} f(x)_{k},
$$

where $T$ is some important set for $G_{P I}$.

Separately, the functions $f(x)$ of the set $f^{\prime}(x)$ are characterized by a specific dependence that highly prepossessed to functional incompleteness and vulnerability with functional deviations from the usual scale or a measure of secondary functional isomorphism in an environment where secondarity is expressed as quantitative qualities without including the main informational purpose (the quin- 
tessence of the function fragment). One way or another the higher organism cannot conduct an adequate functional informational activity, for example, without a nervous system, a classic computer without a storage device, a camera without an optical device, but with no doubt, you can try to hammer a nail or split a nut with this camera.

Thus, it is defining the concept of the universality degree of a state which is in a general sense is comparable to the concept of importance and in a particular sense is inclined to gradual properties i.e. a tendency to functional concentration or specialization or on the contrary a tendency to functional dispersion. A limit $G_{P I}$-type structures specialized on the one hand and common on the other, are mainly expressed depending on the scale in the environment. At the same time, the specialized function, as a special case of the general, is represented in the minimum permissible instance and is the quintessence of the functional information state.

The target or informational affinity of vectors is determining by the measure of the intersection of such vectors in the space of functions. The target similarity of vectors is determined by the degree of the topological identity of such vectors (which is usually accompanied by algorithmic similarity). Thus, the target or informational similarities are not always related but are always similar algorithmically. Consequently, the similarity of structures regulating (which have affinity respectively) states that perform similar functions is the higher, the higher the similarity of the information purposes of such functions. In turn, the similarity of state structures as functions of an informational purpose, which regulating states that perform similar functions, the higher, the higher the similarity of information goals of such functions and so on. In this interpretation, the gradual dependences of the substrate's states similarity and the information goals of their functions are clearly manifested. The similarity of the environment structures seems to depend on the functional-informational similarity of their purposes, where the measure of similarity in both cases is the level of their algorithmic isomorphism.

Some states have special inducible properties when a disturbance, with certain importance, in the structure of state $S$, occurs when there is a special incoming signal $|i(S)|>0$ for $S$ appears, and when $|i(S)|=0$ the signal is the absence of a signal, respectively. All states are inducible. Therefore, we are talking about a special induction component of the same special functionalinformation property of the local state (the most important passing vectors). It is assumed that each state has at least one special inducible attribute or vector.

The state of the $i$-potential for $S$ regulation is the main cause of stability and the general existence of systems in which they belong or the dynamics of the sets which they induce. Such of mechanisms are probably the main and only factor in the formation of coordinated behavior regulation of dissipative systems, which, in turn, determine the dynamics of higher-order sets, etc.

\section{GENERAL DYNAMIC OF SYSTEM}

Each system attempts to establish sustainability, its own independence and integrity on a scale, that forms the limit of visibility or some particular form of emergence $a^{\prime}$ as a contour of a separate system $S$. Even if the dynamics of $S$ looks and behaves according to absolute rest $a^{\prime}(S)$ (not receiving an impulse from out- 
side), there is always an active process $x$ that is part of such a system $x \subset S$ even if such a process is not able to qualitatively affect on $S$ and go beyond the restriction $a^{\prime}$ without external disturbances.

A stable functional state is a consequence, first of all, of the balance stability in the structure of its set repetitions which is formed by functional cycles $f(x)^{*}$ that come from the depths of the state (fig. 1). Deviations $\dagger$ (disturbance appearance) in such cycles determine the balance point shift in the repeat state function $f(x)^{*}$ the functional significance of which is dependent on the stability property of a higher order system. During the system movement in scale, the forming intervals on repetitions acquire differences only in the case of a certain exogenous signal presence.

We can define $N_{n}$ as the set $n \rightarrow \infty$ of depth $D$ in the flow $P$ : $\sum_{k=1}^{n} \alpha_{k}, \beta_{k}, \gamma_{k} \ldots n_{k}=N_{n}$, location of the cyclic function $f(x)$ in a state $S$ can be displayed as $a^{\prime *} \supset \sum_{k=1}^{n}\left(N_{n}^{(*)}\right)_{k} \supset f(x)_{n}^{(*)}$. Fig. 1 shows a functional movement of four states that form a motion vector that transfers a stable state $S_{1}$ to a stable state $S_{2}$ with an unstable structure, where the nested function $f\left(\gamma_{3}^{2}\right)$ receives an incoming destructive signal $i\left(\downarrow_{\gamma}^{2}\right)$, however, depending on the measure of the functional circuit $(a)$ stability that received such a signal is defining its further existence and, consequently, the existence of higher functional circuits $a^{\prime}$ when $a \subset a^{\prime}$. In this case, the function $f\left(\gamma_{3}^{2}\right)$ acts as a property to prevent the disintegration of the cyclic contour $f\left(\gamma_{1-3}^{2}\right)$ even with the change in the function $f\left(\gamma_{3}^{2}\right)$ (the case when an intrafunctional replacement is carried out without a qualitative change in the signal) algorithm and thereby completely leaving the higher structures $f\left(\beta_{1-3}^{2}\right), f\left(\alpha_{1-3}^{2}\right)$ intact. Degenerative functions, depending on their synergistic potential, can initiate the disintegration of the functional state with different speed or efficiency. Formally, fig. 1 displays the dynamics of the action space as a continuous one-dimensional linear set in such a way that this set is a reflection or map of the actions that are identical to the real environment. It should be noted that this interpretation is very compatible with the description of coupled mappings in distribution systems [7].

A destructive signal $i_{d}$ is such if a destructive potential prevails in it and a stabilizing one $i_{s}$ is such if a stabilizing potential prevails in it, respectively. Classical destructive processes are instability, for example, in autowaves, it manifests itself in the formation of turbulence or instability and leading to the rupture of the spirals.

$i_{d}$ can have stabilizing qualities in some local relations while $i_{s}$ destructive, but one way or another $i_{d}$ leads to functional destruction and $i_{s}$ to functional stability in some $N_{n}$, providing there is no effective resistance of the environment that does not enable the distribution $i$ out of $a^{\prime}$. 


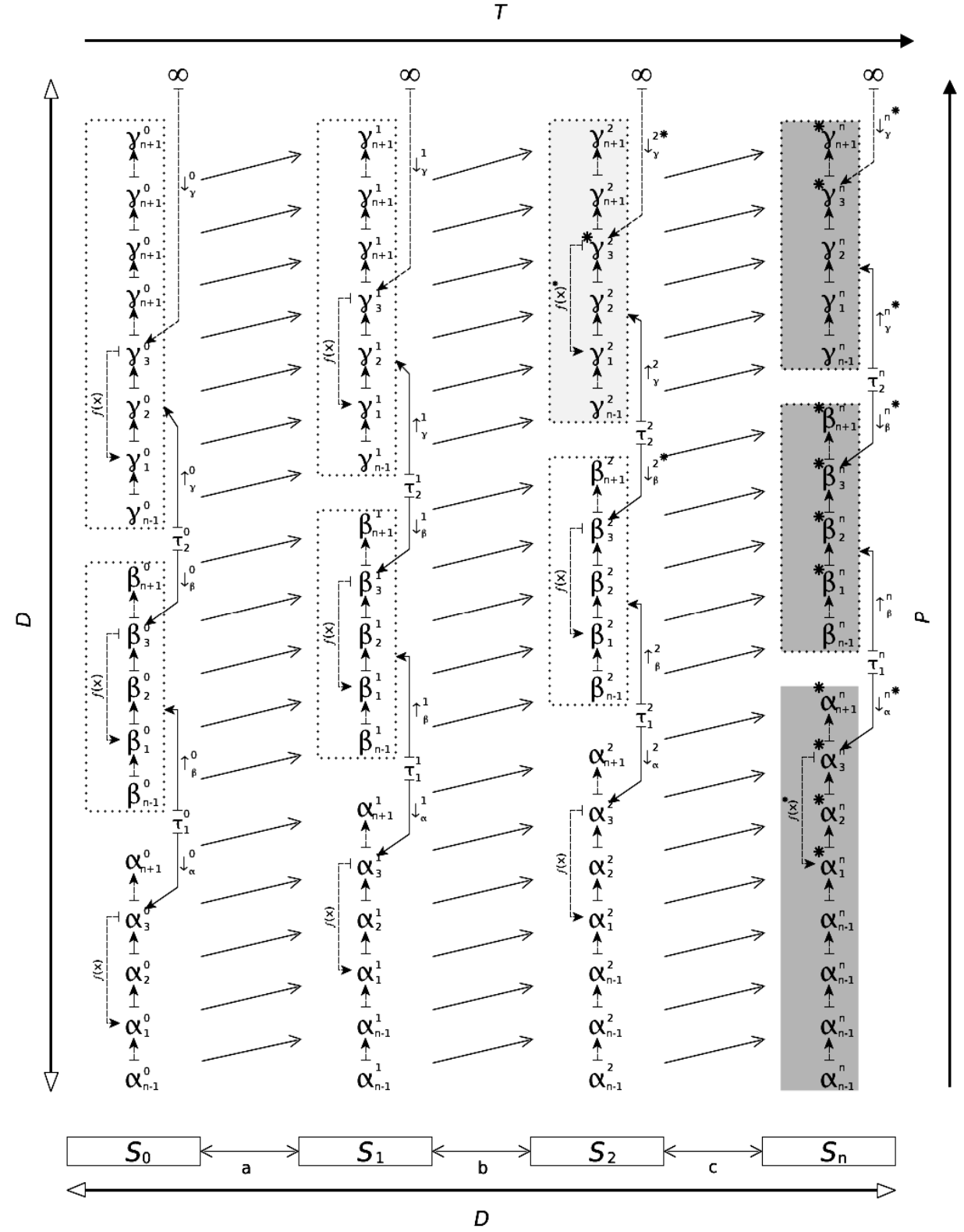

Fig. 1. The diagram illustrates the dynamic features of the functional state in the environmental movement process

The figure displays: $S_{0}, S_{1}, S_{2}, S_{n}$ - are states, when $S_{1}$ is the repeat of $S_{0}$; $S_{2}$ is the iteration of $S_{1}$ etc.; $\alpha, \beta, \gamma, N_{n}$ - sets in the environmental scale, when $\gamma \subset \beta \subset \alpha ; \quad f\left(\alpha_{1-3}\right), f\left(\beta_{1-3}\right), f\left(\gamma_{1-3}\right) \quad$ - ranges of cyclic stable function $P(f(x))=\sum_{k=1}^{n}\left(N_{n(1-3)}\right)_{k}: N_{n} \rightarrow i\left(N_{n}\right)$ - is the sum of all qualitative repeats $f(x)$ of the flow $P$ which retain the original signal; $a, b, c$ - are the depths of actions; $\tau$ - symmetric state (description below); $\uparrow, \downarrow$ - outgoing and incoming functional-information signals; $\alpha_{n}, \beta_{n}, \gamma_{n}$ - noise that is not included in the functional circuit; * - degenerative functions that reduce the stability of the system 
and leading to the rapid disintegration of a stable functional state; $D-$ scale (depth) of the environment; $T$ - direction of the environment repeat generation or states map for the next time; $P-$ flow direction; $\left(D, T, P, a\left(S_{0}, S_{1}\right), b\left(S_{1}, S_{2}\right), c\left(S_{2}, S_{n}\right)\right) \rightarrow \infty$.

The state $S_{1}$ can be considered as a qualitative segment of the state $S_{0}$ repetition, that did not receive $i_{d}$ to some $N_{n}$ of the depth $D$ or if such a signal was received at the "safe" depth then the function that received the signal has full resistance to it. This class of state repetitions seems to have the greater potential for the complex system's formation, the larger interval of $D$ values beyond which the probability in the formation of at least quality destructive signals for the current system $a^{\prime}(D)$ is reduced. For example, the state $S_{2}$ has an incoming destructive signal $i_{d}\left(* \downarrow_{\gamma}^{2}\right)$, that does not have sufficient potential for a qualitative (nonstructural) effect on the function $f\left(\gamma_{3}^{2}\right)$ it happens so that the cyclic function $f\left(\gamma_{1-3}^{2}\right)$ remains completely intact in relation to the higher functional cycle $f\left(\beta_{1-3}^{2}\right)$ and state $S_{2}$ can also be considered as a qualitative repetition of the state $S_{1}$. In turn, the state $S_{n}$ is defined as unstable due to the fact that there is a predominance of self-destructive and not self-stabilizing properties. $i_{d}\left(* \downarrow_{\gamma}^{(2, n)}\right)$, with sufficient potential destroys the functional circuit $f\left(\gamma_{1-3}^{n}\right)$ in such a way that the newly synthesized interval $N_{\gamma-n}$, due to its intact functions (functions that have successfully repeated), still not able to qualitatively affect the $f(\beta, \alpha) \supset f(y)$. At the same time, a similar situation can also occur in a higher function $f\left(\beta_{n}^{n}\right)$ with the only difference that, as a result of a destructive signal $* \downarrow_{\beta}^{n}$, the number of qualitative repetitions in it will not be enough to form a functional circuit $f\left(\alpha_{1-3}^{n}\right)$, which will lead to the complete destruction of the interval on $S$ and the development of a chaotic regime. With the help of a stabilizing feedback signal $i_{S}\left(\uparrow_{\beta}^{n}\right)$ (or a signal having a local stabilizing potential), the function $f\left(\alpha_{n}^{n}\right)$ is quite capable of being qualitatively executed for $S_{n+1}$ until total destruction, when the structure of involved $f\left(\beta_{n}^{n}, \gamma_{n}^{n}\right)$ becomes completely inefficient in ensuring the synthesis of high-quality $S\left(f\left(\alpha_{n}^{n}\right)\right)$ repetition.

The processes of a stable system $N_{S}$, the synthesis of which can be carried out by the intersection of certain cyclic (stable) sets $N_{S}=N_{n} \cap N_{m}$ can also, to some extent, be controlled by states $N_{n m}$ or other processes that somehow involved in the operation of $N_{S}$. That is, "relatives" of a local functional system, the relatedness of which is the higher, the higher their functional predisposition to such a system. Stable $N_{n m}$ type states with their algorithm can also initiate such a stable system $N_{|S+1|}$ that is able to regulatively influence their states-synthesizers $N_{|n m+1|}$. Stable states can be in balance $\uparrow$ both with each other and with the nearest medium and additively maintain stability through initiation and subsequent regu- 
lation of the synthesized states or diffuse recursive regulation of their own functions and other dependent processes in the environment. An indicative and particular example of the described mechanism may be the biochemical functions of the enzymatic intracellular starvation-saturation including extreme cases that lead to the destruction of the system or cell death.

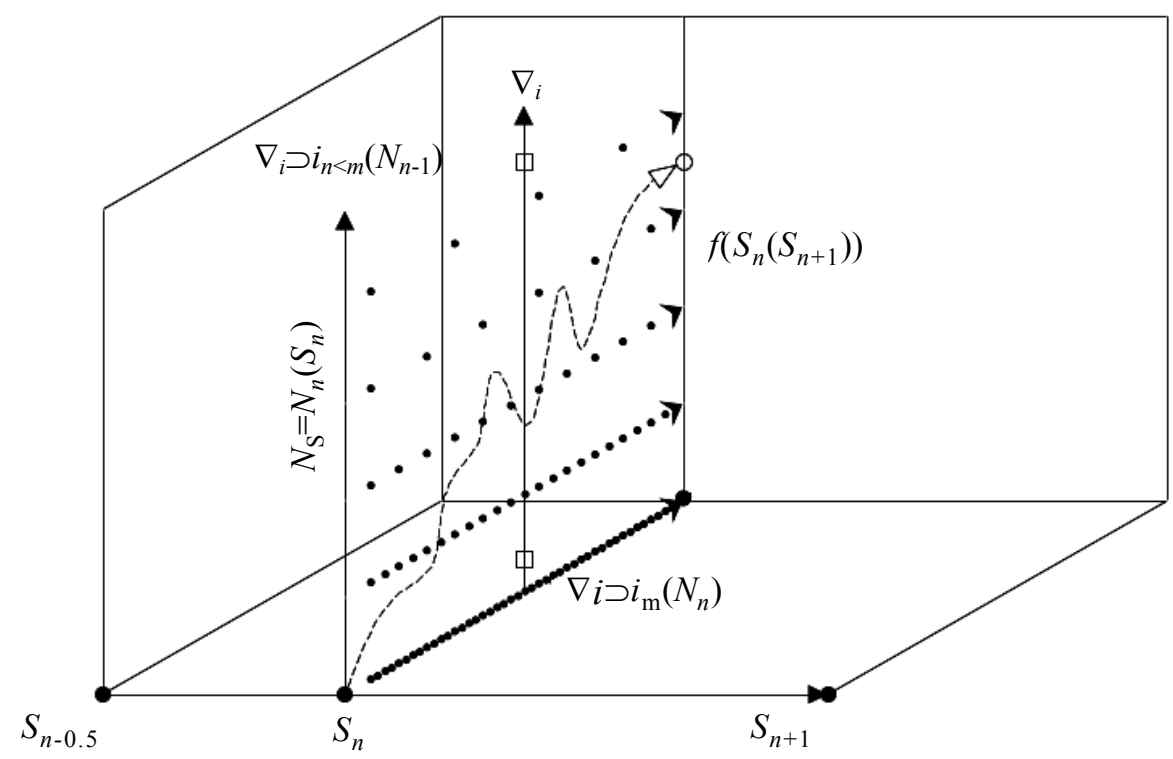

Fig. 2. Dotted arrows schematically indicate the direction of flow in the space of functions, where the depth interval of such a space relatively corresponds to the axis of applicate and indicated by a dotted line with the corresponding density

Processes of individual functional states may have closely related functions or purposes. Previously, in this interpretation, it is worth to characterize the concept of an information gradient (fig. 2) or a changing signal with respect to $N_{n-1}$. This effect is a kind of information "fluctuation" with increasing of the distance from the main function, where the fluctuation is expressed as the dynamic $q$-parameter of the options set $A$ of the function that triggers the signal $i$, when $q=f(A), f(A) \rightarrow i$. In this case, the targets $\nabla i \supset i_{m}\left(N_{n}\right)$ of the initial state have the highest concentration (in the $N$ space) and as the negative scaling proceeds, this functional concentration decreases $\nabla i \supset i_{n<m}\left(N_{n-1}\right)$. The concentration itself is quite similar to the potential function $f$ for the Laplace operator $\frac{\partial^{2} f}{\partial x^{2}}$ on a one-dimensional vector space.

It is obvious that the higher the target $f$ on axis $N_{S}$ from the state (at the point $S_{n}$ ), the less the probability of this state inductions for $f$, which is natural since the number of actions and the entropy factor increase. Consequently, a similar situation arises in a related relation as a special case of $q$-parameter in the space of functions.

In addition, as mentioned above, the vectors of closely related functions intersect, and the measure of the relatedness of such functions depends on a quantity of joint states for such vectors and how far these states from each other. Such constellation intersections can form stable states $N_{n m}$ the number of which becomes dependent on the probability of synthesis a high order steady state $N_{S}^{\prime}$ 
when $N_{S}^{\prime}\left(N_{S}\right) \supset N_{n m} \cdot N_{S}^{\prime}$, in a particular strategy of self-organization, can have reverse stabilizing signals into itself, ensuring sufficient resistance of the $N_{n m}$ to degenerative factors. Thus, it is possible that dependencies running in a specialized probabilistic situation (where the general noise level that including the combined levels of uncertainty constitutes a process of states that contributes to the synthesis and subsequent support for the performance of a certain function) with a certain degree of recursion can be or become stable or important states (such as in the regulation of prokaryotic operons [8]).

We can also highlight the phenomenon of the directional regulation, which is a set of stable states vectors at the intersection of which is synthesizing a deterministic state, which, in turn, with a high probability is also stable due to the predisposition of any regulation to stability in principle. From the effectiveness of regulation, the stability or steadiness of regulation is also determined, since regulation itself can occur only in a stable and steadiness dynamic environment.

\section{EVOLUTION FEATURES}

The phenomenon of affinity may lead to evolutionary regulation and adjustment of indicators of pseudo-probabilistic intersections as the environment moves. In the first case, the signal with a regulating potential is maintained even under the multiple transformations of the initiating state, and in the second case, the regulation of affinity into itself that executed by the function $f(|x|)$.

The ability of complex systems to self-organize and adapt in short-term or long-term (evolutionary) conditions is a natural response to environmental variability. Short-term conditions may correspond in particular to the mechanisms of the Belousov-Zhabotinsky reaction or biological embryonic inductiondifferentiation. In turn, long-term conditions evolutionary phenomena of allogenesis, aromorphosis or anagenesis, etc.

A particular case of long-term self-organization, which seems to have a peak $\uparrow$ importance indicator, is the phenomenon of evolutionary cephalization, that reflecting a significant functional similarity between the individual biological systems of an acceptable, higher taxonomic category and the evolutionary movement under correlation factors over time. An artificially $\uparrow$ induced adaptation phenomenon can be, for example, the domestication of animals with the subsequent restructuring of the organism's genome and the corresponding phenotypic changes, which is also part of the evolutionary self-organization mechanism.

Corresponding factor of the organization complicating that forming evolutionary peaks in the environment, is an increasing the complexity of the nested systems $N_{n+1}$ (on a biological scale, this may be the differentiation of individual cells, their groups, membrane, intracellular and other microsystems), organization that synthesizing corresponded return signals of the external environment $N_{n-1}$ and whose dynamic are also variative. Thus, it is possible to define $i(\tau)$ (see fig. 1) as a symmetric state in a quasi-space $I_{n}$ that inversely complicates the functional-informational structure, which seems to be a crucial part of the evolution strategy in the environment.

The processes of self-organization in all systems and in particular organisms are represented most clearly in the form of protective mechanisms against external and internal influences penetrating the system, increasing the risk of initiating 
destructive progress. In the process of evolution, each organism in any way developed a similar system of protection, more clearly in the form of specific internal biochemical reactions, or more generalized in the form of the strategy features of the whole organism's life cycle or their group. Immune-like processes in evolution, obviously modified in proportion to how the organisms themselves changed their habitat and mainly increased the complexity of the system's organization (for example, CRISPR in prokaryotes, RNA interference (RNAi) in plants, or an immune complex of protection against pathogenic effects in higher organisms including humans).

We can conditionally divide the mechanisms of long-term self-organization formed in evolution into two levels. The first is based on more direct dependencies on the structure of the evolutionary movement, and the second level is forming on the basis of indirect or consequence constellations of the first level or states located closer to the periphery on the gradient plane of functional radiation in the environment. If Immune-like systems belong to the first level of selforganization, then at least all other functionally related processes (that are having a much more significant stochastic origin indicator) can be attributed to the second level of self-organization (for example, the phenomenon of allelic exclusion in the regulation of the immune system B-cells differentiation $[9,10])$. Anyway, each mechanism having obvious signs of any first level self-organization is located closer to the center of the functional radiation pattern, while the indirect or second level is respectively located to the periphery.

\section{REFERENCES}

1. Hénon $M$. The applicability of the third integral of motion: Some numerical experiments / M. Hénon, C. Heiles // The Astronomical Journal. - 1964. — 69. P. 73-79.

2. Campbell K. Protein-Mediated Translation Repression / K. Campbell, J. Beckwith, J. Davies, J. Gallant // Gene Function in Prokaryotes. - New York: Cold Spring Harbor Laboratory, Cold Spring Harbor, 1983. - P. 185-187.

3. Uhlenbeck O.C. Interaction of R17 Coat Protein with Its RNA Binding Site for Translational Repression / O.C. Uhlenbeck, J. Carey, P.J. Romaniok et al. // J. Biomol. Structure and Dynamics. - 1983. - 1. - P. 539--552.

4. Weissbach H. Molecular Mechanisms of Protein Biosynthesis / H. Weissbach, S. Pestka. — New York: Academic Press, 1977. —P. 605-613.

5. Singer M. Ganes and Genomes / M. Singer, P. Berg. — 1991. — P. 514-532.

6. Singer M. Ganes and Genomes / M. Singer, P. Berg. - 1991. — P. 783-799.

7. Loskutov A.Yu. Basics of Complex Systems Theory / A.Yu. Loskutov, A.S. Mihailov. - Izhevsk: Computer research institute, 2007. — P. 393-428.

8. Bauer C. Attenuation in Bacterial Operons. Gene functions in prokaryotes / C. Bauer, J. Carey, L. Kasper. — New York: Cold Spring Habor Laboratory, 1983. P. 65-89.

9. Mostoslavsky $R$. The lingering enigma of the allelic exclusion mechanism / R. Mostoslavsky, W. Alt Frederick, K. Rajewsky // Cell. — 2004. Vol. 118, Issue 5. - P. 539-544.

10. Liang Hong-Erh. Variegated transcriptional activation of the immunoglobulin kappa locus in pre-b cells contributes to the allelic exclusion of light-chain expression / Liang Hong-Erh, Hsu Lih-Yun, Cado Dragana, S. Schlissel Mark // Cell. 2004. - Vol. 118, Issue 1. - P. 19-29.

Received 10.04.2019

From the Editorial Board: the article corresponds completely to submitted manuscript. 